\title{
Rigorously Bayesian Range Finder Sensor Model for Dynamic Environments
}

\author{
Tinne De Laet, Joris De Schutter and Herman Bruyninckx
}

\begin{abstract}
This paper proposes and experimentally validates a Bayesian network model of a range finder adapted to dynamic environments. The modeling rigorously explains all model assumptions and parameters, improving the physical interpretation of all parameters and the intuition behind the model choices. With respect to the state of the art model [1], this paper proposes: (i) a different functional form for the probability of range measurements caused by unexpected objects, (ii) an intuitive explanation for the discontinuity encountered in the cited paper, and (iii) a reduction in the number of model parameters, while maintaining the same representational power for experimentally obtained data. The proposed beam model is called RBBM, short for Rigorously Bayesian Beam Model. A maximum-likelihood estimation and a variational Bayesian estimation algorithm (both based on expectation-maximization) are proposed to learn the model parameters.
\end{abstract}

\section{INTRODUCTION}

Intelligent robot behavior requires sensors to allow a robot to perceive its environment. To translate sensor measurements into intelligent behavior, they have to be interpreted in the context of a measurement model that describes the physical formation process of a measurement. The design of a measurement model is a trade-off between accuracy (hence, increased intelligence) on the one hand, and complexity and robustness on the other hand. The latter are improved by models that use only physically interpretable parameters. Accuracy has two aspects: (i) the mathematical measurement model is a good approximation of the physical sensor, and (ii) the stochastic nature of the physics involved is represented by conditional probabilities on a well-chosen subset of the model parameters.

This paper focuses on (sonar and laser) range finders, whose physical principle is the emission of a sound or light wave, followed by the recording of its echo. Highly accurate measurement models would include physical phenomena such as surface curvature and material absorption coefficient, but these are difficult to estimate robustly in unstructured environments. Hence, the literature typically relies on purely geometric models, either in discrete geometric grids [2]-[6], or in continuous geometric models [1], [7], [8]. Moravec [6] proposed (in general) non-Gaussian measurement densities over a discrete grid of possible distances measured by sonar; the likelihood of the measurements has to be computed for

All authors gratefully acknowledge the financial support by K.U.Leuven's Concerted Research Action GOA/05/10 and the Research Council K.U.Leuven, CoE EF/05/006 Optimization in Engineering (OPTEC). Tinne De Laet is a Doctoral Fellow of the Fund for Scientific Research-Flanders (F.W.O.) in Belgium.

The authors are with the Department of Mechanical Engineering, Katholieke Universiteit Leuven, Celestijnenlaan 300B, B-3001 Leuven, Belgium. Tinne. DeLaet@mech. kuleuven.be all possible positions of the mobile robot at a certain time. Even simplified models [5] in this approach turned out to be computationally too expensive for real time, so [4] proposed a sensor model which makes use of only the distance to the closest obstacle in the map along the direction of the sensor; the model is a mixture of two physical causes for a measurement: a hit with an object in the map, or with an object not yet modeled in the map. An analogous mixture [1], [7] adds two more physical causes: a "random" measurement, and a "maximum reading" measurement resulting from using the sensor beyond its range. [1], [8] use a continuous model, [7] presents the discrete analog of the mixture, taking into account the limited resolution of the range sensor.

This paper proposes a rigorously Bayesian modeling of the probabilistic measurement model for a range finder adapted for the use in dynamic environments. Similar to [1], [8] the measurement model is derived for the continuous domain. Unlike the previous proposed models the mixture components are founded on a rigorously Bayesian modeling. This modeling makes use of probabilistic graphical models, in this case Bayesian networks. The proposed beam model is called RBBM, short for Rigorously Bayesian Beam Model.

\section{BeAm Models of RAnge Finders}

The goal of this paper is to derive a probabilistic measurement model $p(Z=z \mid X=x, M=m)^{1}$ for a range finder, i.e. the beam model; $Z$ indicates the measured range, $X$ the position of the mobile robot (and of the sensor mounted on it), and $M$ the environment map. An ideal deterministic range sensor gives a range measurement $z^{\star}=g(x, m)$, $g()$ being the ideal measurement function. In practice, ideal measurements are disturbed by, for instance, physical noise and inaccurate modeling of sensor and environment. The probabilistic representation $p(z \mid x, m)$ of the measurement model reflects the uncertain outcome of the measurement due to such disturbances. Since the environment is often assumed static, unmodeled dynamics in the environment (e.g., people or unmodeled robots moving in front of the sensor) directly jeopardize the estimation results.

This paper gives a rigorously Bayesian derivation for a probabilistic measurement model of range finders in a dynamic environment. The innovations of the presented approach are (i) to introduce extra state variables $A=a$ for the positions of unmodeled objects in the probabilistic measurement model $p(z \mid x, m, a)$, and (ii) to marginalize

\footnotetext{
${ }^{1}$ To simplify notation, the explicit mention of the random variable in the probabilities $p(X=x)$ is omitted whenever possible, and replaced by the common abbreviation $p(x)$.
} 
out these extra state variables from the total probability before estimation. This is required because extra variables (exponentially!) increase the computational complexity of state estimation, and the position of unmodeled objects is typically not of great interest anyway. In summary, the marginalization

$$
p(z \mid x, m)=\int_{a} p(z \mid x, m, a) p(a) d a,
$$

avoids the increase in complexity to infer the conditional probability distributions $p(x)$ and $p(m)$, while maintaining the modeling of the dynamic nature of the environment. Section II-A explains which extra state variables are physically relevant; Section II-B explains the steps to simplify the large joint probability density function of the extended state to a factored form that is computationally cheap to marginalize.

\section{A. Bayesian model with extra state variables}

Bayesian networks graphically represent probabilistic relationships among the variables in a mathematical model, to structure probabilistic inference computations with those variables [9], [10]. The network consists of: (i) A set of random variables connected by directed edges forming a directed acyclic chain (DAG); (ii) Each discrete (continuous) random variable has a finite (infinite) set of mutually exclusive states; (iii) each random variable $A$ with parents $B_{1}, \ldots, B_{N}$ has a conditional probability distribution $p\left(A \mid B_{1}, \ldots, B_{n}\right)$ (called conditional probability table for discrete variables). In this application, an unknown number $n$ of unmodeled objects is present in the environment. Depending on the position of the $j$ th unmodeled object on the measurement beam $x_{N j}$, the unmodeled object is occluding the environment or not. $k$ is the total number of occluding objects out of the $n$ unmodeled objects, and the position of such occluding objects on the measurement beam is denoted by $x_{K i}$. If the environment is occluded by an unmodeled object, the range sensor will ideally measure $z_{\mathrm{occl}}^{\star}=x_{K c}$, with $x_{K c}$ the position of the closest occluding object.

The following extra state variables $a$ of Eq. (1) are included in the Bayesian model: $N$ is the discrete random variable indicating the unknown number of unmodeled objects in the environment; $X_{N j}$ is the continuous random variable for the position of the $j$ th unmodeled object on the measurement beam; $K$ is the discrete random variable indicating the number of objects occluding the measurement of the map; $X_{K i}$ is the continuous random variable for the position of the $i$ th occluding object on the measurement beam; and $Z_{\text {occl }}^{\star}$ is the continuous random variable indicating the ideal range measurement of a the closest occluding object. Figure 1 shows all these variables $Z, X, M, N, X_{N}=$ $\left\{X_{N j}\right\}_{j=1: n}, K, X_{K}=\left\{X_{K i}\right\}_{i=1: k}, Z_{\text {occl }}^{\star}$ in the Bayesian network for the probabilistic range finder measurement model.

The arrows in the network model causal relationships between the variables. For example, $X$ and $M$ unambiguously determine the measured range $Z$ in the case of a perfect sensor and in absence of unmodeled occluding objects.

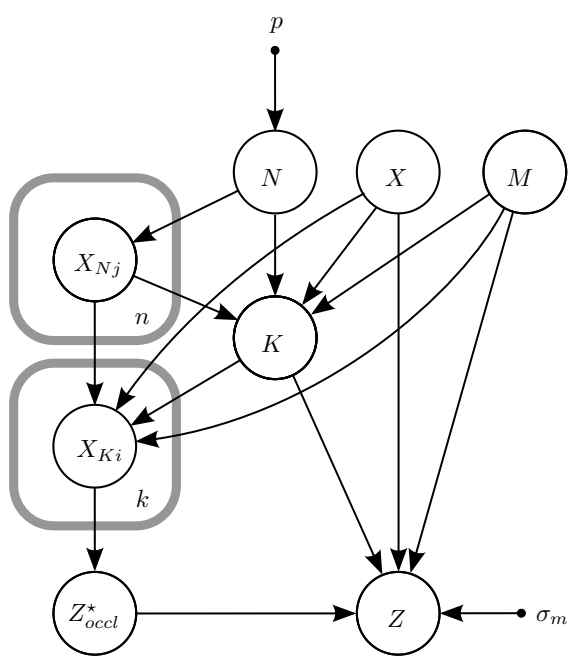

Fig. 1. The Bayesian network for the probabilistic measurement model supplemented with the deterministic parameters shown explicitly by the smaller solid nodes using a compact representation with plates (the rounded rectangular boxes). A plate represents a number, indicated in the lower right corner, of independent nodes of which only a single example is shown explicitly.

The number of occluding objects $K$ depends on the total number $N$ of unmodeled objects and their positions $X_{N}$ with respect to the measurement beam. Also $X$ and $M$ have a causal impact on $K$ : the larger the expected measurement $z^{\star}$, the higher the possibility that one or more objects are occluding it. The positions on the measurement beam $X_{K}$ of the occluding objects are equal to the positions of the $K$ unmodeled objects that are occluding the map. Therefore, the random variables $X_{K}$ are not only influenced by $K$ but also by $X_{N}$. Since the $K$ objects are occluding the map, their positions on the measurement beam are limited to the interval $\left[0, z^{\star}\right]$, so $X_{K}$ has a causal dependency on $X$ and $M$. The ideal measurement $z_{\mathrm{occl}}^{\star}$ of an occluding object is the position of the occluding object closest to the sensor, so $Z_{\text {occl }}^{\star}$ depends on the positions $X_{K}$ of the occluding objects. Finally, the measurement $Z$ depends also on the ideal measurement of the occluding object $Z_{\mathrm{occl}}^{\star}$ and the number of occluding objects $K$.

As explained before inferring the probability distribution of the extra state variables such as $p(n)$ is often infeasible. Marginalization of the extra state variables $Z, X, M, N, X_{N}, K, X_{K}, Z_{\text {occl }}^{\star}$ avoids the increase in complexity of the estimation problem, but maintains the modeling of the dynamic nature of the environment. Marginalization requires all conditional probability tables and conditional probability distributions of each random variable conditionally on its parents. Therefore, first of all some assumptions have to be made for $p(n)$. Assume that the probability of the number of unmodeled objects decreases exponentially, i.e. $p(n)$ is given by:

$$
p(n)=(1-p) p^{n},
$$

with $p$ defined here as the degree of appearance of unmodeled 
objects. Secondly, assume that nothing is known a priori about the position of the unmodeled objects on the measurement beam, so the unmodeled object's position is assumed to be uniformly distributed over the measurement beam:

$$
p\left(x_{N j} \mid n\right)=\left\{\begin{array}{ll}
\frac{1}{z_{\max }} & \text { if } x_{N j} \leq z_{\max } \\
0 & \text { otherwise }
\end{array} .\right.
$$

Thirdly, assume the unmodeled objects are independent:

$$
p\left(x_{N} \mid n\right)=\prod_{j=1}^{n} p\left(x_{N j} \mid n\right) .
$$

Next, an expression is needed for the conditional probability: $p\left(k \mid n, x_{N}, x, m\right)$, i.e. the probability that $k$ of the $n$ unmodeled objects are occluding the measurement of the map $m$. An unmodeled object is occluding the map $m$ if it is lying on the measurement beam and in front of the map feature. It can be easily seen (or mathematically derived), that $p\left(k \mid n, x_{N}, x, m\right)$ is a binomial distribution:

$p\left(k \mid n, x_{N}, x, m\right)= \begin{cases}\left(\begin{array}{l}n \\ k\end{array}\right) \mu^{k}(1-\mu)^{n-k} & \text { if } k \leq n \\ 0 & \text { otherwise }\end{cases}$

where $\mu$ is the probability that an unmodeled object is occluding the map and $\left(\begin{array}{l}n \\ k\end{array}\right)=\frac{n !}{(n-k) ! k !}$ is the number of ways of choosing $k$ objects out of a total of $n$ objects. Since it was assumed that the positions of the unmodeled objects were uniformly distributed $\mu$, the probability that an unmodeled object is occluding the map is:

$$
\mu=p\left(x_{N j}<z^{\star}\right)=\frac{z^{\star}}{z_{\max }} .
$$

Furthermore, an analytical expression for $p\left(x_{K} \mid x_{N}, k\right)$ is necessary. The positions of the occluding objects $x_{K}$ are the positions of those unmodeled objects $x_{N}$ that are occluding the environment. In other words, $x_{K i}$ equals $x_{N j}$ if and only if the unmodeled object is occluding the environment, i.e. $x_{N j} \leq z^{\star}$ :

$$
\begin{aligned}
& p\left(x_{K i} \mid x_{N j}, k, x, m\right)= \\
& \left\{\begin{array}{cl}
\frac{1}{p\left(x_{N j} \leq z^{\star}\right)} \delta\left(x_{K i}-x_{N j}\right) & \\
=\frac{z_{\max }}{z^{\star}} \delta\left(x_{K i}-x_{N j}\right) & \text { if } x_{N j} \leq z^{\star} \\
0 & \text { otherwise }
\end{array}\right.
\end{aligned}
$$

with $\delta$ the Dirac function and $x_{K i}$ the occluding object corresponding to $x_{N j}$. The perfect measurement of a occluding object $z_{\mathrm{occl}}^{\star}$ is that of the closest occluding object $x_{K c}$ :

$$
p\left(z_{\text {occl }}^{\star} \mid x_{K}\right)=\delta\left(z_{\text {occl }}^{\star}-x_{K c}\right) .
$$

The conditional probability $p\left(z \mid x, m, z_{\text {occl }}^{\star}, k\right)$ has two main cases, the first for $k=0$ where no occlusion is present and the sensor is observing the modeled map $m$ and the second case for $k \geq 1$ where the sensor observes an occluding object. If the measurement noise is modeled by a zero mean Gaussian with standard deviation $\sigma_{m}$,

$$
p\left(z \mid x, m, z_{\mathrm{occl}}^{\star}, k\right)= \begin{cases}\mathcal{N}\left(z ; z^{\star}, \sigma_{m}\right) & \text { if } k=0 \\ \mathcal{N}\left(z ; z_{\mathrm{occl}}^{\star}, \sigma_{m}\right) & \text { if } k \geq 1 .\end{cases}
$$

The Bayesian network for the probabilistic measurement model supplemented with the introduced parameters $p$ and $\sigma_{m}$ is shown in Figure 1.

\section{B. Marginalization of extra state variables}

This section shows the different steps needed to marginalize out the extra state variables introduced in Eq. (1), and motivates the assumptions that lead to an analytical measurement model.

The product rule rewrites the measurement model $p(z \mid x, m)$ as:

$$
p(z \mid x, m)=\frac{p(z, x, m)}{p(x, m)}=\frac{p(z, x, m)}{p(x) p(m)},
$$

since $X$ and $M$ are independent. The numerator is obtained by marginalizing the joint probability of the whole Bayesian network $p_{\text {joint }}=p\left(z, x, m, n, x_{N}, k, x_{K}, z_{\text {occl }}^{\star}\right)$ over $z_{\text {occl }}^{\star}$, $x_{K}, x_{N}, n$ and $k$ :

$p(z, x, m)=\int \sum_{k} \int \sum_{n} \int p_{\text {joint }} d x_{N} d x_{K} d z_{\text {occl }}^{\star}$

Using the chain rule to factorize the joint distribution and substituting (11) in (10) gives:

$$
\begin{array}{r}
p(z \mid x, m)=\int \sum_{k} p\left(z \mid x, m, z_{\mathrm{occl}}^{\star}, k\right) \int p\left(z_{\mathrm{occl}}^{\star} \mid x_{K}\right) \\
p\left(k, x_{K} \mid x, m\right) d x_{K} d z_{\mathrm{occl}}^{\star},
\end{array}
$$

$$
\begin{aligned}
& \text { where } p\left(k, x_{K} \mid x, m\right)=\sum_{n} p(k \mid n, x, m) p(n) \\
& \underbrace{\int_{x_{N}} p\left(x_{K} \mid x_{N}, k, x, m\right) p\left(x_{N} \mid n\right) d x_{N}}_{=p\left(x_{K} \mid n, k, x, m\right)},
\end{aligned}
$$

in which the binomial distribution $p\left(k \mid n, x_{N}, x, m\right)$ of Eq. (5) can be moved out of the integral (since it is independent of $\left.x_{N}\right)$ and is further on written as $p(k \mid n, x, m)$. Now focus on the integral over $x_{N}$ and study the part over the position of the object $x_{N j}$. Substituting (3) and (7) results in:

$$
p\left(x_{K i} \mid n, k, x, m\right)= \begin{cases}\frac{1}{z^{\star}} & \text { if } x_{K i} \leq z^{\star} \\ 0 & \text { otherwise. }\end{cases}
$$

This shows that $x_{K i}$ is uniformly distributed when conditioned on $k, n$. Since all occluding objects are considered independent:

$$
p\left(x_{K} \mid n, k, x, m\right)= \begin{cases}\left(\frac{1}{z^{\star}}\right)^{k} & \text { if } x_{K i} \leq z^{\star}, \forall 0 \leq i \leq k \\ 0 & \text { otherwise. }\end{cases}
$$

Substituting the above equation in (13) gives:

$$
p\left(k, x_{K} \mid x, m\right)= \begin{cases}\left(\frac{1}{z^{\star}}\right)^{k} p(k \mid x, m) & \text { if }\left\{\begin{array}{l}
x_{K i} \leq z^{\star} \\
\forall 0 \leq i \leq k
\end{array}\right. \\
0 & \text { otherwise }\end{cases}
$$


where $\left(1 / z^{\star}\right)^{k}$ is moved out of the integral and

$$
p(k \mid x, m)=\sum_{n} p(k \mid n, x, m) p(n) .
$$

Substituting (2) and (5) in the above infinite sum over $n$ gives:

$p(k \mid x, m)=\sum_{n=k}^{\infty}\left[\left(\begin{array}{c}n \\ k\end{array}\right) \mu^{k}(1-\mu)^{n-k}(1-p) p^{n}\right]$.

It can be proved that this infinite sum can be simplified to:

$$
p(k \mid x, m)=\left(1-p^{\prime}\right) p^{\prime k} \text {, with } p^{\prime}=\frac{\mu p}{1-(1-\mu) p} .
$$

As will be seen later on, $p^{\prime}$ has a clear physical interpretation: it is the probability that the map is occluded by an unmodeled object (Eq. 38). Substituting the analytical expression for $p(k \mid x, m)$ in (16) and substituting the result in (12) gives:

$$
\begin{aligned}
p(z \mid x, m) & =\int \sum_{k} p\left(z \mid x, m, z_{\mathrm{occl}}^{\star}, k\right)\left(1-p^{\prime}\right) p^{\prime k} \\
p\left(z_{\text {occl }}^{\star} \mid k, x, m\right) d z_{\mathrm{occl}}^{\star}, \quad \text { with } & \int_{x_{K}} p\left(z_{\mathrm{occl}}^{\star} \mid x_{K}\right) p\left(x_{K} \mid k\right) d x_{K} .
\end{aligned}
$$

Substituting (8) in the above equation results in:

$$
\begin{aligned}
p\left(z_{\text {occl }}^{\star} \mid k, x, m\right) & =\int_{x_{K c}} \delta\left(z_{\text {occl }}^{\star}-x_{K c}\right) p\left(x_{K c} \mid k\right) d x_{K c} \\
& =p\left(x_{K c}=z_{\text {occl }}^{\star} \mid k, x, m\right) .
\end{aligned}
$$

This equation shows that the conditional probability $p\left(z_{\text {occl }}^{\star} \mid k, x, m\right)$ represents the probability that the perfect measurement of the nearest occluding object is $z_{\text {occl }}^{\star}$, i.e. the probability that the nearest occluding object is located at $z_{\mathrm{occl}}^{\star}$. This is only the case when one of the $k$ objects on the measurement beam is located such that $z_{\text {occl }}^{\star}$ is measured and all other objects on the measurement beam are located behind the occluding object, or expressed in probabilities:

$$
\begin{aligned}
& p\left(z_{\mathrm{occl}}^{\star} \mid k, x, m\right)= \\
& \sum_{i=1}^{k} p\left(x_{K \neq i} \geq z_{\mathrm{occl}}^{\star} \mid k, x, m\right) p\left(x_{K i}=z_{\mathrm{occl}}^{\star} \mid k, x, m\right) .
\end{aligned}
$$

Since $x_{K}$ is uniformly distributed over $\left[0, z^{\star}\right]$ (14), $p\left(z_{\text {occl }}^{\star} \mid k, x, m\right)$ can be written as:

$$
p\left(z_{\text {occl }}^{\star} \mid k, x, m\right)=k \frac{1}{z^{\star}}\left(\frac{z^{\star}-z_{\text {occl }}^{\star}}{z^{\star}}\right)^{k-1} .
$$

Now turn the attention to the summation over $k$ in (20):

$$
\begin{aligned}
& p\left(z, z_{\mathrm{occl}}^{\star} \mid x, m\right)= \\
& \sum_{k} p\left(z \mid x, m, z_{\mathrm{occl}}^{\star}, k\right) p\left(z_{\mathrm{occl}}^{\star} \mid k, x, m\right)\left(1-p^{\prime}\right) p^{\prime k} .
\end{aligned}
$$

Split the summation in (25) in two parts: one for $k=0$, when there is no occlusion and one for $k \geq 1$ when there is, and substitute the expression for $p\left(z \mid x, m, z_{\text {occl }}^{\star}, k\right)$ given by (9):

$$
\begin{gathered}
p\left(z, z_{\mathrm{occl}}^{\star} \mid x, m\right)=\mathcal{N}\left(z ; z^{\star}, \sigma_{m}\right) p\left(z_{\mathrm{occl}}^{\star} \mid k=0, x, m\right) \\
\left(1-p^{\prime}\right)+\mathcal{N}\left(z ; z_{\mathrm{occl}}^{\star}, \sigma_{m}\right) p\left(z_{\mathrm{occl}}^{\star} \mid x, m\right),
\end{gathered}
$$

with $p\left(z_{\mathrm{occl}}^{\star} \mid x, m\right)=\sum_{k=1}^{\infty} p\left(z_{\mathrm{occl}}^{\star} \mid k, x, m\right)\left(1-p^{\prime}\right) p^{\prime k}$.

Substituting (24) in (27) results in:

$p\left(z_{\text {occl }}^{\star} \mid x, m\right)=\sum_{k=1}^{\infty} k \frac{1}{z^{\star}}\left(\frac{z^{\star}-z_{o c c l}^{\star}}{z^{\star}}\right)^{k-1}\left(1-p^{\prime}\right) p^{\prime k}$,

which can be simplified to:

$$
p\left(z_{\text {occl }}^{\star} \mid x, m\right)=\frac{\left(1-p^{\prime}\right) p^{\prime}}{z^{\star}\left[1-\left(\frac{z^{\star}-z_{o c c l}^{\star}}{z^{\star}} p^{\prime}\right)\right]^{2}} .
$$

Substituting (29) in (26) gives:

$$
\begin{gathered}
p\left(z, z_{\mathrm{occl}}^{\star} \mid x, m\right)=\mathcal{N}\left(z ; z^{\star}, \sigma_{m}\right) p\left(z_{\mathrm{occl}}^{\star} \mid k=0, x, m\right) \\
\left(1-p^{\prime}\right)+\mathcal{N}\left(z ; z_{\mathrm{occl}}^{\star}, \sigma_{m}\right) \frac{\left(1-p^{\prime}\right) p^{\prime}}{z^{\star}\left[1-\left(\frac{z^{\star}-z_{\mathrm{occl}}^{\star}}{z^{\star}} p^{\prime}\right)\right]^{2}} .
\end{gathered}
$$

Substituting the result of the summation over $k$ in (20) shows that only the integration over $z_{\text {occl }}^{\star}$ still has to be carried out:

$$
\begin{aligned}
& p(z \mid x, m)=\left(1-p^{\prime}\right) \mathcal{N}\left(z ; z^{\star}, \sigma_{m}\right)+p^{\prime} \\
& \quad \int \mathcal{N}\left(z ; z_{\mathrm{occl}}^{\star}, \sigma_{m}\right) \frac{1-p^{\prime}}{z^{\star}\left[1-\left(\frac{z^{\star}-z_{\text {ocl }}^{\star}}{z^{\star}} p^{\prime}\right)\right]^{2}} d z_{\mathrm{occl}}^{\star} .
\end{aligned}
$$

The first term of the right hand side is a Gaussian distribution around the ideal measurement multiplied with the probability of no occlusion $(k=0)$. The second term is an integration over all possible positions of the occluding object of a scaled Gaussian distribution centered at the ideal measurement of the occluding object $\left(z_{\text {occl }}^{\star}\right)$. The scaling factor represents the probability the occluding objects are located such that $z_{\text {occl }}^{\star}$ is measured:

$$
p\left(z_{\mathrm{occl}}^{\star} \mid x, m\right)=\frac{p^{\prime}\left(1-p^{\prime}\right)}{z^{\star}\left[1-\left(\frac{z^{\star}-z_{\text {occl }}^{\star}}{z^{\star}} p^{\prime}\right)\right]^{2}} .
$$

This scaling factor can be rewritten as:

$$
p\left(z_{\text {occl }}^{\star} \mid x, m\right)=\frac{p(1-p)}{z_{\max }\left[1-\left(1-\frac{z_{\text {occl }}^{\star}}{z_{\max }}\right) p\right]^{2}},
$$

which is independent of $z^{\star}$. This is in accordance with intuition, since one expects $p\left(z_{\text {occl }}^{\star} \mid x, m\right)$ i.e. the probability distribution of the ideal measurements caused by the occluding objects to be independent of $z^{\star}$, the measurement in the case there would be no occlusion. Furthermore, the likelihood of sensing unexpected objects decreases with the range, as expected. This is easily explained with the following thought experiment: if two independent objects are 


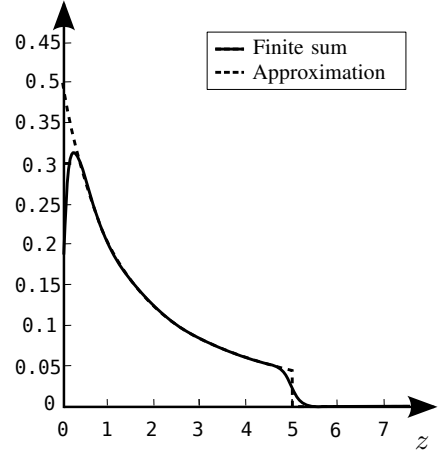

Fig. 2. Quality of approximation of integral with $p=0.8, z_{\max }=10$, $z^{\star}=5$ and $\sigma_{m}=0.15$.

present with the same likelihood in the perception field of the range finder, and the first object is closest to the range sensor, then it is more likely to measure the first object. To measure the second object, the second object should be present and the first object should be absent [1].

Until now, no approximations where made to obtain Eq. (31) for the beam model $p(z \mid x, m)$. The integral over the scaled Gaussian distributions however, can't be obtained analytically. Therefore a first approximation in the marginalization is made, neglecting the noise on the range measurement in the case an occluded object is measured, i.e.: $\mathcal{N}\left(z ; z_{\mathrm{occl}}^{\star}, \sigma_{m}\right) \approx \delta\left(z-z_{\mathrm{occl}}^{\star}\right)$, where $\delta$ represents the Dirac function. Using this approximation the integral in the right hand side of (31) is approximated as:

$$
\frac{p^{\prime}\left(1-p^{\prime}\right)}{z^{\star}\left[1-\frac{z^{\star}-z}{z^{\star}} p^{\prime}\right]^{2}}=\frac{p(1-p)}{z_{\max }\left[1-\left(1-\frac{z}{z_{\max }}\right) p\right]^{2}} .
$$

Figure 2 shows the quality of the approximation compared to the approximation of the integral by an finite sum with step size 0.01. It is clear that the approximation introduces a discontinuity around $z=z^{\star}$. Using the proposed approximation for the integral the resulting measurement function is: $p(z \mid x, m)=$

$$
\begin{cases}\left(1-p^{\prime}\right) \mathcal{N}\left(z ; z^{\star}, \sigma_{m}\right)+p^{\prime} \frac{\left(1-p^{\prime}\right)}{z^{\star}\left[1-\left(\frac{z^{\star}-z}{z^{\star}} p^{\prime}\right)\right]^{2}} & \text { if } z \leq z^{\star} \\ \left(1-p^{\prime}\right) \mathcal{N}\left(z ; z^{\star}, \sigma_{m}\right) & \text { otherwise }\end{cases}
$$

as shown in Figure 3.

The measurement model can be written as a mixture of two components:

$$
\begin{aligned}
p(z \mid x, m) & =\pi_{1} p_{\text {hit }}(z \mid x, m)+\pi_{2} p_{\text {occl }}(z \mid x, m), \\
\text { with } \pi_{1} & =\left(1-p^{\prime}\right) \\
\pi_{2} & =p^{\prime} \\
p_{\text {hit }}(z \mid x, m) & =\mathcal{N}\left(z ; z^{\star}, \sigma_{m}\right) \\
p_{\text {occl }}(z \mid x, m) & = \begin{cases}\frac{1}{z^{\star}} \frac{1-p^{\prime}}{\left[1-\left(\frac{z^{\star}-z}{z^{\star}} p^{\prime}\right)\right]^{2}} & \text { if } 0 \leq z \leq z^{\star} \\
0 & \text { otherwise. }\end{cases}
\end{aligned}
$$

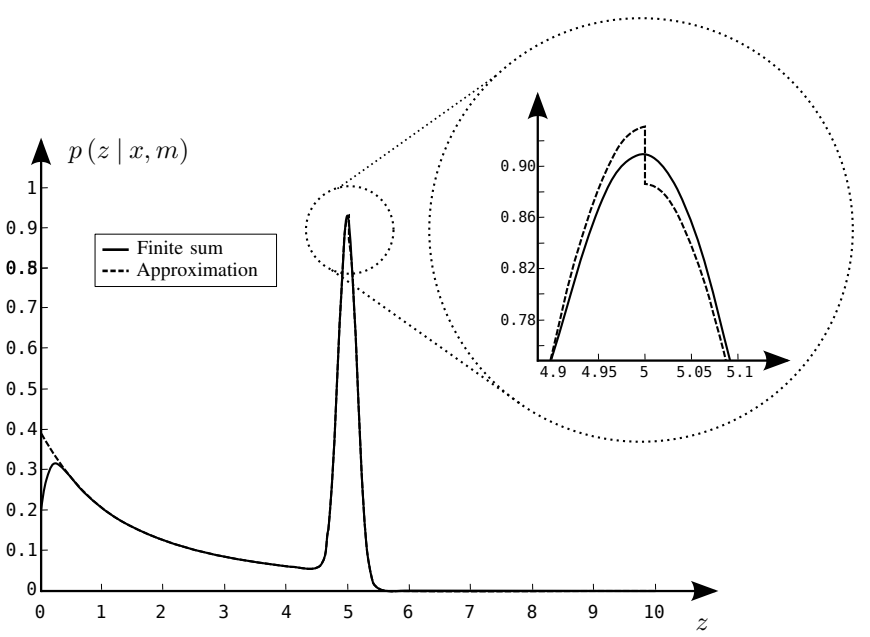

Fig. 3. $p(z \mid x, m)$ with $p=0.8, z_{\max }=10, z^{\star}=5$ and $\sigma_{m}=0.15$.

\section{Extra components}

Occasionally, range finders produce entirely unexplainable measurements. These unexplainable measurements are often caused by phantom readings when sonars bounce off walls, or suffer from cross-talk [1]. These unexplainable measurements are modeled using a uniform distribution spread over the entire measurement range $\left[0, z_{\max }\right]$ :

$$
p_{\text {rand }}(z \mid x, m)= \begin{cases}\frac{1}{z_{\max }} & \text { if } 0 \leq z \leq z_{\max } \\ 0 & \text { otherwise. }\end{cases}
$$

Furthermore, sensor failures typically produce max-range measurements, modeled as a point-mass distribution centered around $z_{\max }$ :

$$
p_{\max }(z \mid x, m)=I\left(z_{\max }\right)= \begin{cases}1 & \text { if } z=z_{\max } \\ 0 & \text { otherwise. }\end{cases}
$$

These two extra components can be added to (36), resulting in the final RBBM:

$$
\begin{aligned}
p(z \mid x, m) & =\pi_{1} p_{\text {hit }}(z \mid x, m)+\pi_{2} p_{\text {occl }}(z \mid x, m)+ \\
& \pi_{3} p_{\text {rand }}(z \mid x, m)+\pi_{4} p_{\max }(z \mid x, m),
\end{aligned}
$$

where $\pi_{3}$ and $\pi_{4}$ are the probabilities that the range finder returns an unexplainable measurement and a maximum reading, respectively. Furthermore,

$$
\begin{aligned}
& \pi_{1}=\left(1-p^{\prime}\right)\left(1-\pi_{3}-\pi_{4}\right) \text { and } \\
& \pi_{2}=p^{\prime}\left(1-\pi_{3}-\pi_{4}\right),
\end{aligned}
$$

while $p_{\text {hit }}(z \mid x, m), \quad p_{\text {occl }}(z \mid x, m), \quad p_{\text {rand }}(z \mid x, m)$ and $p_{\max }(z \mid x, m)$ are given by (39), (40), (41) and (42) respectively.

With respect to the state of the art beam model of Thrun et al. [1], the model proposed here, Eq. (43), has: (i) a different functional form for the probability of range measurements caused by unmodeled objects, (ii) an intuitive explanation for the discontinuity encountered in the cited paper, and (iii) a reduction in the number of model parameters. Thrun 


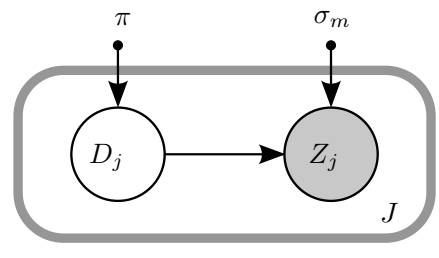

Fig. 4. Graphical representation of the mixture measurement model with latent correspondance variable and intrinsic parameters.

et al. [1] assume that $p_{\text {occl }}(z \mid x, m)$ has an exponential distribution, while this paper shows that the distribution is quadratic, Eq. (40). As stated in the previous paragraph, the discontinuity of the RBBM (Fig. 3) is caused by an approximation. While Thrun's model considers the decay of $p_{\text {occl }}(z \mid x, m)$ to be independent of $\pi_{2}$, the probability of an occlusion, it is shown here that they both depend on the same parameter $p^{\prime}$ (Eq. (40), Eq. (45)). Therefore the RBBM has fewer parameters than Thrun's model.

\section{Learning the RBBM Model Parameters}

The RBBM, Eq. (43), depends on four independent model parameters:

$$
\Theta=\left[\sigma_{m}, p^{\prime}, \pi_{3}, \pi_{4}\right],
$$

while $z_{\max }$ is a known sensor characteristic. This set of parameters has a clear physical interpretation; $\sigma_{m}$ is the standard deviation of the zero mean Gaussian measurement noise in Eq. (9) governing $p_{\text {hit }}(z \mid x, m)$ (Eq. (39)); $p^{\prime}$, defined in Eq. (19), is the probability that the map is occluded $(p(k \geq 1 \mid x, m)) ; \pi_{3}$ and $\pi_{4}$ are the probabilities that the range finder returns an unexplainable measurement (unknown cause) and a maximum reading (sensor failure), respectively.

The physical interpretation of the $\Theta$ parameters allows to initialize them by hand. However, another, more flexible way is to learn the model parameters from actual data. Furthermore, this is also a validation for the proposed analytical model: if the learning algorithm succeeds in finding model parameters such that the resulting distribution gives a good explanation of the data, the analytical model is likely to agree well with reality. This section presents a maximum likelihood (ML) and a variational Bayes (VB) estimator to learn the parameters from data.

\section{A. Maximum likelihood estimator}

A ML estimator is proposed to identify the parameters $\Theta$ that maximize the likelihood of the data $Z=\left\{z_{j}\right\}$ with associated positions $X=\left\{x_{j}\right\}$ and map $m$ :

$$
\Theta=\arg \max _{\Theta} \log p(Z \mid X, m, \Theta) .
$$

When using the mixture representation of the measurement model $(43)^{2}$ the estimation problem can be formulated as

\footnotetext{
${ }^{2}$ In the proposed estimators the maximum reading component is not considered. Extension of the estimators to include this extra component is however straightforward.
}

finding the ML estimates for the parameters $\pi_{1}, \pi_{2}, \pi_{3}$ and $\pi_{4}$. The ML estimate for the parameter $p^{\prime}$ is easily obtained from the reformulated ML estimates using $p^{\prime}=\frac{\pi_{2}}{1-\pi_{3}-\pi_{4}}$. Since it is not known which of the three possible causes actually caused each of the measurements under consideration the ML estimation problem is difficult and lacks a closedform solution. If however, the corresponding causes of the datapoints would be known, the solution is easily obtained in closed form. Therefore introduce a latent correspondence variables $D$ having a 1 -of-K representation in which a particular element $d_{k}$ is equal to 1 and all other elements are zero. This indicates that the cause $k$ caused the datapoint under consideration. The graphical representation of the mixture formulation of the measurement model including the latent correspondence variable is shown in Figure 4. Although with this unknown correspondences, the ML estimation problem lacks a closed-form solution, an expectation-maximization approach (EM) can solve the problem by iterating an expectation and a maximization step. The EM-algorithm is an elegant and powerful method for finding ML solutions for models with latent variables [11]-[13]. The expectation step calculates an expectation for the correspondence variables $d_{k}$ while the maximization step computes the other model parameters under these expectations. Algorithm 1 presents an EM-approach for the ML-estimator, further on called MLEM algorithm.

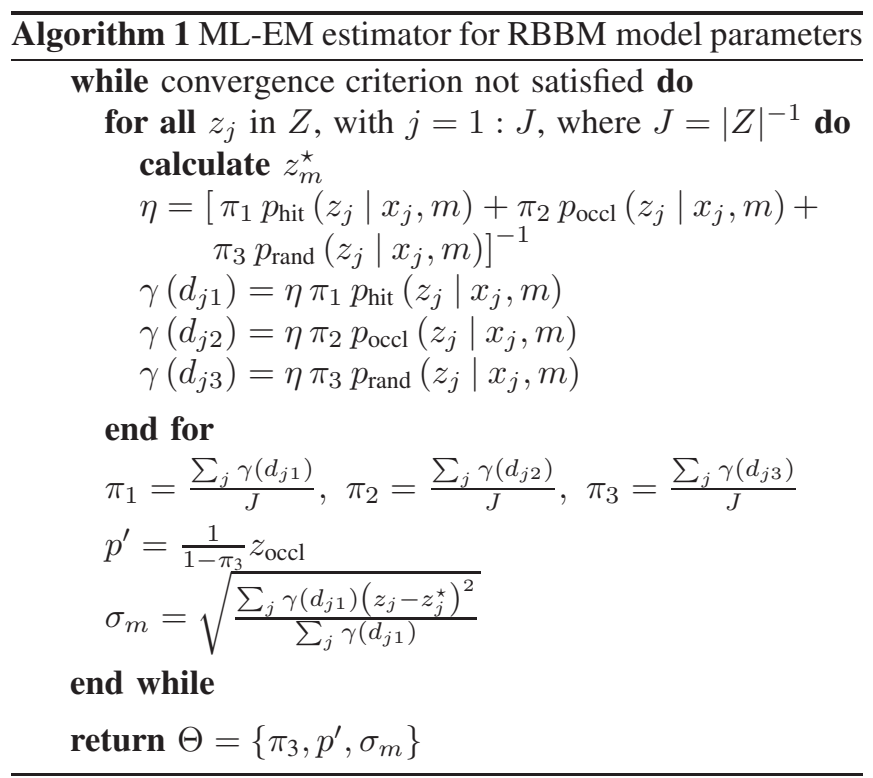

\section{B. Variational inference}

ML approaches give a point estimate of the parameters such that these parameters maximize the likelihood of the data. A well known problem with ML methods is that the likelihood function will generally be higher for more complex models, leading to overfitting [13]-[15]. Fully Bayesian treatment, including a prior over the unknown parameters, automatically overcomes the problems of the ML approach, such as overfitting and give a full probability distribution over the estimated parameters instead of a point estimate. 
The proper Bayesian approach attempts to integrate over the possible settings of all uncertain quantities rather than optimize them as in the ML approach [14], [15]. The quantity that results from integrating out both the hidden variables and the parameters is termed the marginal likelihood: $p(z)=$ $\int p(z \mid \theta) p(\theta) d \theta$, where $p(\theta)$ is a prior over the parameters of the model. Integrating out parameters penalizes models with more degrees of freedom since these models can a priori model a larger range of data sets. This property of Bayesian integrations had been called Occam's razor, since it favors simpler explanations for the data over complex ones [16], [17].

Unfortunately the marginal likelihood, $p(z)$, is an intractable quantity to compute for almost all models of interest. The variational Bayesian method constructs a lower bound on the marginal likelihood, and attempts to optimize this bound using an iterative scheme that has intriguing similarities to the standard EM-algorithm. To emphasize the similarity with ML-EM the algorithm based on variational Bayesian inference is often called VB-EM.

Since the VB approach is a fully Bayesian approach, priors have to be introduced over the parameters $\theta=\left[\mu, \sigma_{m}, \pi\right]$. Since the analysis is considerably simplified if conjugate prior distributions are used, a Dirichlet prior is chosen for the mixing coefficients $\pi: p(\pi)=\operatorname{Dir}\left(\pi \mid \alpha_{0}\right)$, and an independent Gaussian-Wishart prior for the mean $\mu$ and the precision $\lambda_{m}=\sigma_{m}^{-1}$ of the Gaussian distribution $p_{\text {hit }}(z \mid z, m)$ : $p\left(\mu, \lambda_{m}\right)=\mathcal{N}\left(\mu \mid m_{0},\left(\beta \lambda_{m}\right)^{-1}\right) \mathcal{W}\left(\lambda_{m} \mid W_{0}, \nu_{0}\right) . \alpha_{0}$ gives the effective prior number of observations associated with each component of the mixture. Therefore, if the value of $\alpha_{0}$ is set small, the posterior distribution will be mainly influenced by the data rather than by the prior.

Algorithm 2 presents the algorithm for the VB-estimator, further on called VB-EM algorithm.

\section{VALIDATION AND EXPERIMENTS}

In a learning experiment, the parameters of the measurement model, $p(z \mid x, m)$, are learned from data obtained using a laser distance sensor both using the proposed MLEM and VB-EM estimator. In the experiment, range finder measurements are obtained using a static and known map in which unmodeled and possibly moving objects are randomly and manually placed in the field of view of the laser range finder. The laser range finder is a Sick LMS 200 and is located at a fixed position in the known map. From the obtained data set measurements are selected which are centered around a particular expected range. The experiment set consists of eight experiments, with different degree of appearance $p$ (Eq. (2)) of unmodeled objects and different expected ranges.

The model parameters are learned for eight experiments, with different degree of appearance of unmodeled objects. The difference between the learned model and the data is calculated as:

$$
d=\Delta z \sqrt{\sum_{i}\left(p\left(Z^{\star}(i) \mid X(i), m, \Theta\right)-N_{z}(i)\right)^{2}},
$$

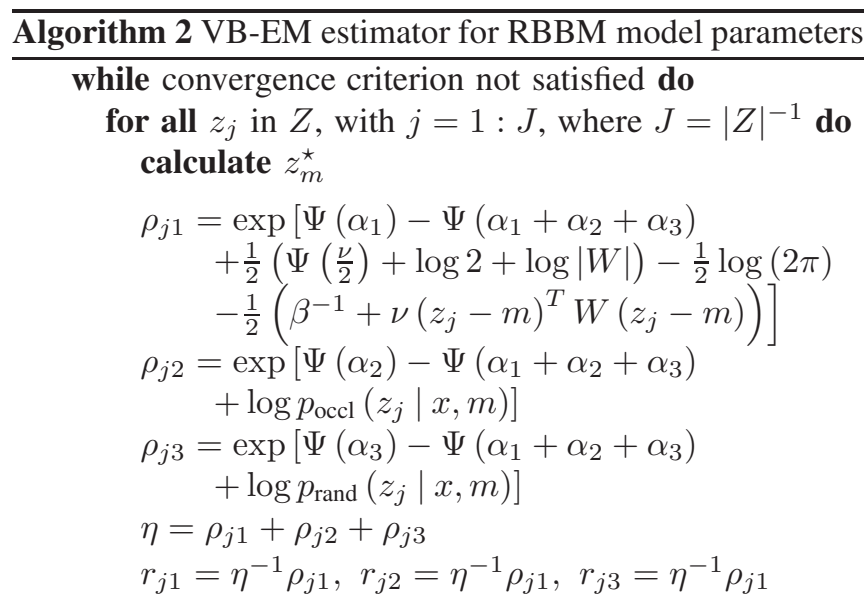

\section{end for}

$$
\begin{aligned}
& J_{1}=\sum_{j=1}^{J} r_{j 1}, J_{2}=\sum_{j=1}^{J} r_{j 2}, J_{3}=\sum_{j=1}^{J} r_{j 3} \\
& \bar{z}_{1}=\frac{1}{J_{1}} \sum_{j=1}^{J} r_{j 1} z_{j} \\
& S_{1}=\frac{1}{J_{1}} \sum_{j=1}^{J} r_{j 1}\left(z_{j}-\bar{z}_{1}\right)\left(z_{j}-\bar{z}_{1}\right)^{T}, \\
& \alpha_{1}=\alpha_{0}+J_{1}, \alpha_{2}=\alpha_{0}+J_{2}, \alpha_{3}=\alpha_{0}+J_{3} . \\
& \beta=\beta_{0}+J_{1} \\
& m=\frac{1}{\beta}\left(\beta_{0} m_{0}+J_{1} \bar{z}_{1}\right) \\
& W^{-1}=W_{0}^{-1}+J_{1} S_{1}+\frac{\beta_{0} J_{1}}{\beta_{0}+J_{1}}\left(\bar{z}_{1}-m_{0}\right)\left(\bar{z}_{1}-m_{0}\right)^{T} \\
& \nu=\nu_{0}+J_{1} \\
& \pi_{1}=\frac{\alpha_{1}}{\alpha_{1}+\alpha_{2}+\alpha_{3}}, \pi_{2}=\frac{\alpha_{2}}{\alpha_{1}+\alpha_{2}+\alpha_{3}}, \pi_{3}=\frac{\alpha_{3}}{\alpha_{1}+\alpha_{2}+\alpha_{3}} \\
& p^{\prime}=\frac{\pi_{2}}{1-\pi_{3}} \\
& \sigma_{m}=\left(\frac{\nu \beta}{1+\beta} W\right)^{-\frac{1}{2}}
\end{aligned}
$$

\section{end while}

return $\left\{\alpha_{1}, \alpha_{2}, \alpha_{3}, \beta, m, W, \nu, \Theta=\left[\pi_{3}, p^{\prime}, \sigma_{m}\right]\right\}$

where $N_{z}$ contains the experiment data as a normalized histogram for the points $Z^{\star}=\left[0: \Delta z: z_{\max }\right]$ :

$$
N_{z}=\frac{\operatorname{hist}\left(Z, Z^{\star}\right)}{\Delta z \sum \operatorname{hist}\left(Z, Z^{\star}\right)} .
$$

In the VB-EM algorithm a low informative prior is used, i.e. $\alpha_{0}$ is low compared to the size of the data set used for learning.

Table I contains the distances for the ML-EM and VB-EM estimator proposed in the paper compared to the distances for the maximum likelihood estimator for the measurement model proposed in [1] for the eight experiments. Despite the extra parameter of the model presented in [1] compared to the model presented here, the average distance between the data and the learned distributions is almost equal. This results indicate that the extra parameter is not needed in the measurement model.

Figure 5 shows the results of the ML-EM and VBEM estimators for the measurement model proposed in the paper compared to the results of the maximum likelihood 


\begin{tabular}{c|ccc} 
Experiment & ML-EM & VB-EM & Model [1] \\
\hline 1 & 0.1272 & 0.1905 & 0.2102 \\
2 & 0.4561 & 0.3518 & 0.4579 \\
3 & 0.5980 & 0.6617 & 0.5115 \\
4 & 0.2404 & 0.2523 & 0.2148 \\
5 & 0.3268 & 0.3414 & 0.3874 \\
6 & 0.4395 & 0.4293 & 0.5259 \\
7 & 0.4286 & 0.4107 & 0.5216 \\
8 & 0.4521 & 0.4411 & 0.3825 \\
\hline average & 0.3849 & 0.3848 & 0.4015 \\
& \multicolumn{2}{|c}{ TABLE I }
\end{tabular}

TABLE I

THE DISTANCES TO THE TRAINING SET OF A ML-EM AND VB-EM ESTIMATOR PROPOSED IN THE PAPER COMPARED TO THE DISTANCES OF A ML-EM ESTIMATOR PROPOSED IN [1] FOR EIGHT EXPERIMENTS.

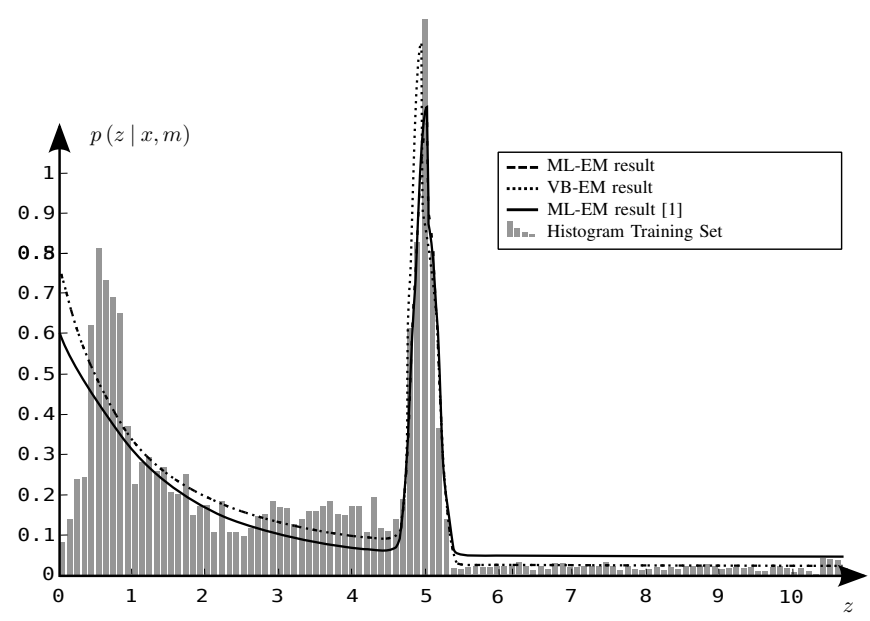

Fig. 5. Results of a ML-EM and VB-EM estimators proposed in the paper compared to the results of a ML-EM estimator proposed in [1] for one of the eight learning experiments with high degree of appearance of unmodeled objects.

estimators for the measurement model proposed in [1] for one of the eight experiments with high degree of appearance of unmodeled objects.

\section{Discussion}

This paper proposed and experimentally validated the RBBM, a rigorously Bayesian network model of a range finder adapted to dynamic environments. The rigorous modeling revealed all underlying assumptions and parameters. This way a clear physical interpretation of all parameters is obtained which provides intuition for the parameter choices. In contrast to [1] the assumption underlying the non-physical discontinuity in the measurement model is discovered. Furthermore, it is shown that the functional form for the probability of range measurements caused by unmodeled objects $p_{\text {hit }}(z \mid x, m)$ (43) is quadratic rather than exponential as proposed in [1], [7], [8]. Furthermore, compared to [1],
[7], [8] the number of parameters is reduced, while still allowing the same representational power for experimentally obtained data. Bayesian modeling revealed that both the ramp of $p_{\text {hit }}(z \mid x, m)$ and the probability of an occluded measurement $\pi_{2}$ are directly dependent on one parameter $p^{\prime}$. This is in contrast with the measurement models proposed so far, where these two parameters are assumed to be independent. Finally, a maximum-likelihood estimation and a variational Bayesian estimation algorithm both based on the expectation-maximization approach were proposed to learn the RBBM model parameters. Learning the model parameters directly benefits from the obtained reduction in number of parameters. Using eight learning experiments it was shown that the RBBM is able to explain the obtained measurements as good as a state of the art model [1].

\section{REFERENCES}

[1] S. Thrun, W. Burgard, and D. Fox, Probabilistic Robotics. MIT Press, 2005.

[2] D. Hähnel, D. Schulz, and W. Burgard, "Mobile robot mapping in populated environments and sensor planning," Journal of the Advanced Robotics, vol. 17, no. 7, pp. 579-597, 2003.

[3] D. Hähnel, R. Triebel, W. Burgard, and S. Thrun, "Map building with mobile robots in dynamic environments," in Proceedings of the 2003 IEEE International Conference on Robotics and Automation, Taipeh, Taiwan, 2003, pp. 1557-1569.

[4] D. Fox, W. Burgard, and S. Thrun, "Markov localization for mobile robots in dynamic environments," Journal of Artificial Intelligence Research, vol. 11, pp. 391-427, 1999.

[5] W. Burgard, D. Fox, D. Hennig, and T. Schmidt, "Estimating the absolute position of a mobile robot using position probability grids," in Proc. of the National Conference on Artificial Intelligence, 1996.

[6] H. P. Moravec, "Sensor fusion in certainty grids for mobile robots," AI Magazine, vol. 9, pp. 61-74, 1988.

[7] H. Choset, K. M. Lynch, S. Hutchinson, G. A. Kantor, W. Burgard, L. E. Kavraki, and S. Thrun, Principles of Robot Motion: Theory, Algorithms, and Implementations. MIT Press, June 2005.

[8] P. Pfaff, W. Burgard, and D. Fox, "Robust monte-carlo localization using adaptive likelihood models," in European Robotics Symposium, H. Christensen, Ed., vol. 22. Palermo, Italy: Springer-Verlag Berlin Heidelberg, Germany, March 2006, pp. 181-194.

[9] F. V. Jensen and T. D. Nielsen, Bayesian Networks and Decision Graphs. Springer, 2007.

[10] R. E. Neapolitan, Learning Bayesian Networks. New York, NY: Pearson Prentice Hall, 2004.

[11] A. P. Dempster, N. M. Laird, and D. B. Rubin, "Maximum likelihood from incomplete data via the EM algorithm (with discussion)," Journal of the Royal Statistical Society (Series B), vol. 39, pp. 1-38, 1977.

[12] G. J. McLachlan and T. Krishnan, The EM algorithm and extensions. New York, NY: John Wiley \& Sons, 1997.

[13] C. M. Bishop, Pattern Recognition and Machine Learning. Springer, 2006.

[14] M. J. Beal, "Variational algorithms for approximate Bayesian inference," Ph.D. dissertation, University College London, 2003.

[15] M. J. Beal and Z. Ghahramani, "The variational bayesian em algorithm for incomplete data: with application for scoring graphical model structures," in Valencia International Meeting on Bayesian Statistics, Tenerife, Canary Islands, Spain, 2003.

[16] W. Jeffreys and J. Berger, "Ockham's razor and bayesian analysis," American Scientist, vol. 80, pp. 64-72, 1992.

[17] C. Rasmussen and Z. Ghahramani, "Occam's razor," in Advances in Neural Information Processing 13. Denver, Colorado: MIT Press, December 2000. 